\title{
NON-LINEAR MODE COUPLING IN SYMMETRICALLY KINKED BARS
}

\author{
K. A. LEGGE. \\ Department of Physics, University of New England, Armidale, New South Wales 2351, Australia \\ AND \\ N. H. Fletcher \\ Institute of Physical Sciences, CSIRO, Limestone Avenue, Canberra ACT 2602, Australia
}

(Received 9 October 1986, and in revised form 11 December 1986)

\begin{abstract}
A theoretical and experimental study of energy transfer between the vibrational modes of a symmetrically kinked bar with clamped ends is described. Two non-linear mechanisms responsible for energy transfer from one mode to another at twice the frequency are identified. The first arises from the interaction between shear and tensional forces at the kink and the second from unbalanced moments across the kink. In the system studied, the first of these mechanisms is dominant. A further related mechanism is responsible for energy transfer to modes at three times the base frequency. When a kinked bar with mode frequencies of a few hundred hertz is shaped so that two modes have the desired 2 to 1 frequency relation and is excited by striking it at a node of the higher mode, then the amplitude of that mode rises from zero to a maximum in a time of order $0.1 \mathrm{~s}$ and then decays. Theory and experiment are in quite good agreement in relation to the time delay to the maximum amplitude and the magnitude of this maximum. The results contribute to an understanding of the vibrational behaviour of certain musical gongs and are also relevant to other systems.
\end{abstract}

\section{INTRODUCTION}

The linear vibrational behaviour of strings, bars, plates and shells is well understood, although the complexity of the analysis increases as we progress through this series. Also well understood and documented is the non-linear "hardening" behaviour, with increasing amplitude, of the vibrations of strings, bars and flat plates clamped rigidly at their edges $[1,2]$. The situation for shells is more complex and may result in either hardening or softening behaviour depending on thickness and curvature [3] but again the basic theory is well understood.

There is, however, another effect of non-linearity observed in such systems when the boundary conditions are a little more complicated which results in a coupling between modes and a transfer of energy from those initially excited to others which may be completely missing from the original motion. In certain musical gongs designed to exploit this effect there may be a nearly complete transfer of vibrational energy from low frequency to high frequency modes over a time of order one second, giving a pronounced audible effect [4]. The phenomenon is, however, of more general and fundamental interest than this particular example might suggest.

As a first step towards understanding this non-linear mode coupling and energy transfer, we began by demonstrating and analyzing the phenomenon for a flexible string passing 
over a non-rigid bridge [5]. In that system both frequency doubling and frequency tripling were observed and, since its mode frequencies are harmonic to a good approximation, energy transfer to missing modes on a timescale of about $0 \cdot 1$ seconds was observed and accounted for by the theory.

The present paper represents the second step in this development and examines related behaviour in simply kinked bars, the kink being found to be essential to the process if simple clamped boundary conditions are assumed. A simple symmetrically kinked bar was chosen for the study, partly for reasons of simplicity and partly because it represents an appropriate progression between a string passing over a bridge and a gong in the form of a plate bounded by a conical flange. The phenomena discussed, however, have more general occurrence.

The situation studied is shown in Figure 1, the bar being symmetrically kinked at points $B$ and $B^{\prime}$ and rigidly clamped at its extreme ends, $C$ and $C^{\prime}$. We concentrate our attention on symmetric modes to reduce the algebra-antisymmetric modes can be treated similarly and there is no coupling between the two classes. We shall find that the essence of the non-linearity is the amplitude-dependent tension generated in the bar by its motion and the coupling of this tension to shear forces at the kinks $B$ and $B^{\prime}$, though there is a related but less significantly non-linearity associated with coupling to the bending moments.

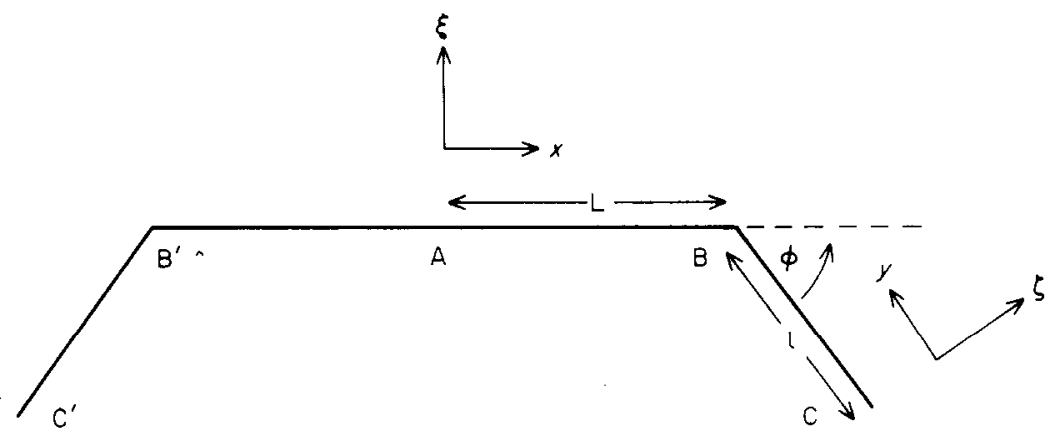

Figure 1. The kinked bar and its co-ordinate system.

\section{LINEAR THEORY}

As our method of analysis looks at the effects of non-linear forces on the linear modes of the bar, it is necessary first to calculate the linear solution to the equation of motion of the bar. In the simplest linear approximation, the equation of motion for any straight section of bar may be written

$$
\rho \partial^{2} \xi / \partial t^{2}=-Q \kappa^{2} \partial^{4} \xi / \partial x^{4}+\left(T_{1} / S\right) \partial^{2} \xi / \partial x^{2}-D \partial \xi / \partial t,
$$

where $\xi$ is the normal displacement, $\rho$ is the density of the material, $S$ is the cross-sectional area, $\kappa$ is the radius of gyration, $Q$ is Young's modulus, $D$ is a measure of damping and $T_{1}$ is the tension in the bar. With this co-ordinate system adopted for the section AB of the bar with length $L$, a simple change of notation gives the equation of motion for the bent end $\mathrm{BC}$ with length $l$,

$$
\rho \partial^{2} \zeta / \partial t^{2}=-Q \kappa^{2} \partial^{4} \zeta / \partial y^{4}+\left(T_{2} / S\right) \partial^{2} \zeta / \partial y^{2}-D \partial \zeta / \partial t
$$

where $\zeta$ is the normal displacement and $T_{2}$ is the tension along this section. The co-ordinates are defined in Figure 1. 
For simplicity only modes symmetric about the midpoint of the bar will be considered. Symmetry then requires the following conditions at point A:

$$
\partial \xi / \partial x=0, \quad \partial^{3} \xi / \partial x^{3}=0 \quad \text { at } \quad x=0 .
$$

The solution to equation ( 1 ) is therefore

$$
\xi_{n}(x)=\left[a_{n} \cos \alpha_{n} x+b_{n} \cosh \alpha_{n} x\right] \sin \left(\omega_{n} t+\phi_{n}\right) \exp \left(-t / \tau_{n}\right),
$$

where $a_{n}$ and $b_{n}$ are constants dependent only on further matching conditions at point $\mathrm{B}, \omega_{n}$ is the mode frequency, and $\tau_{n}$ is derived from the damping coefficient $D$.

Boundary conditions at the clamped end $C$, namely

$$
\zeta-0, \quad \partial \zeta / \partial y=0 \quad \text { at } \quad y=0
$$

give the solution to equation (2) as

$$
\begin{aligned}
\zeta(y)= & {\left[A_{n}\left(\sin \alpha_{n} y-\sinh \alpha_{n} y\right)+B_{n}\left(\cos \alpha_{n} y-\cosh \alpha_{n} y\right)\right] } \\
& \times \sin \left(\omega_{n} t+\phi_{n}\right) \exp \left(-t / \tau_{n}\right)
\end{aligned}
$$

where again the constants $A_{n}$ and $B_{n}$ depend on matching conditions at point $B$. One can assume for the linear approximation that $T_{1}=T_{2}=0$ so that, in both equations (4) and (6),

$$
\alpha_{n}=\left[\omega_{n}^{2} \rho / Q \kappa^{2}\right]^{1 / 4} .
$$

Matching conditions at the point $B$ can now be used to determine the relation between $a_{n}, b_{n}$ and $A_{n}, B_{n}$ and also the mode frequency $\omega_{n}$ and decay time $\tau_{n}$. For continuity at $B$ one must equate the components of displacement and write quite generally, as illustrated in Figure 2, with $Z_{1}, Z_{2}$ for $\xi(L), \zeta(l)$ respectively, these quantities being negative as drawn,

$$
Z_{1}=-\delta \sin \phi+Z_{2} \cos \phi, \quad-\Delta=\delta \cos \phi+Z_{2} \sin \phi .
$$

$\Delta$ and $\delta$ are the displacements parallel to the $x$ and $y$ axes respectively, arising out of second-order changes in length of the bar and may therefore be equated to zero in first order. This reduces equations $(8)$ to

$$
Z_{1}=Z_{2} \cos \phi, \quad 0=Z_{2} \sin \phi
$$

so that, provided $\phi \neq 0$, then $Z_{1}=Z_{2}=0$, and point $\mathrm{B}$ remains fixed. Furthermore since bending moments at $B$ must be finite and continuous,

$$
\partial \xi /\left.\partial x\right|_{x=L}=-\partial \zeta /\left.\partial y\right|_{y=l}, \quad \partial^{2} \xi /\left.\partial x^{2}\right|_{x=L}=\partial^{2} \zeta /\left.\partial y^{2}\right|_{y=i} .
$$

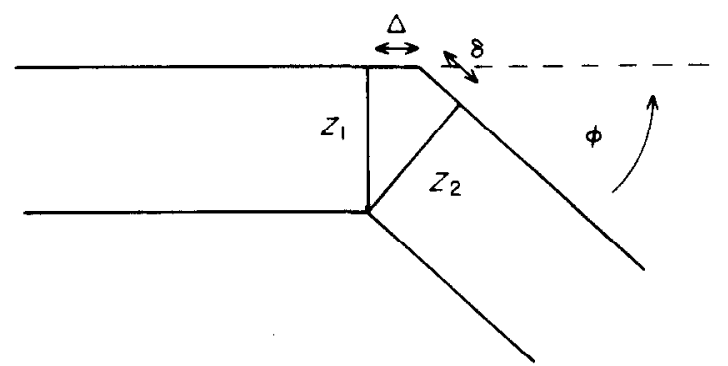

Figure 2. Second-order displacements at the kink. $\Delta$ and $\delta$ are positive and $Z_{1}, Z_{2}$ negative as drawn. 


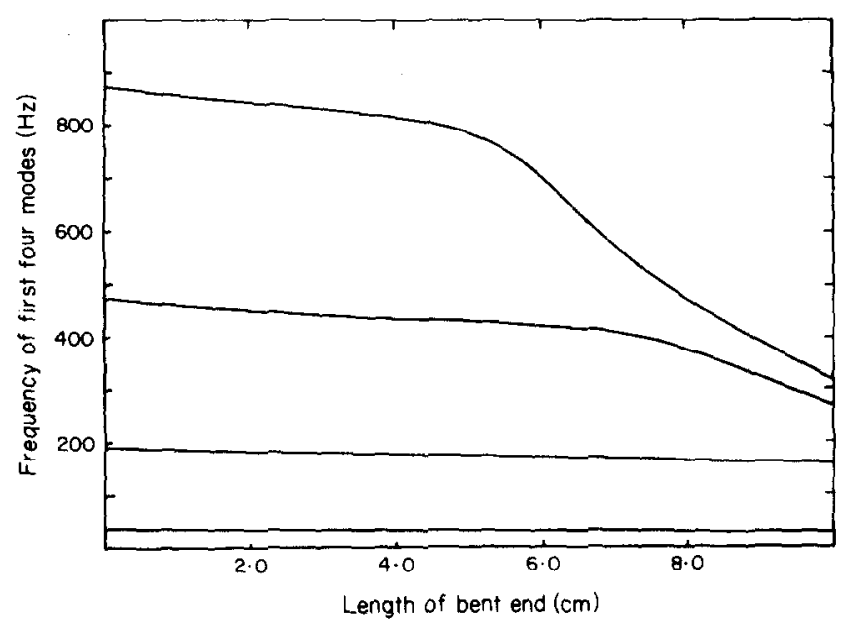

Figure 3. Frequencies of the symmetric modes of a kinked bar with central length $30 \mathrm{~cm}$ and thickness $0.6 \mathrm{~mm}$ as the length $l$ of the clamped end sections is varied.

These conditions are sufficient to relate $a_{n}, b_{n}, A_{n}$ and $B_{n}$ and to produce an equation relating the frequencies $\omega_{n}$ of the vibrational modes to the lengths $L$ and $l$ of the bar sections. Figure 3 is a plot of the variation of the frequencies of the first four symmetric modes with the length $l$ of the section $\mathrm{BC}$ while $L$, the length of section $\mathrm{AB}$, remains constant. These modes are, of course, orthogonal.

\section{NON-LINEAR FORCING TERMS}

As suggested earlier, there are two separate non-linear forcing terms to be considered. The first, and as it turns out the more important one, arises from the coupling of tension and shear forces of the bar sections. Figure 4 shows the shear and tensile forces present on the bar sections $\mathrm{AB}$ and $\mathrm{BC}$. Balance of forces at the bend $\mathrm{B}$ requires

$$
F_{1}+F_{2} \cos \phi-T_{2} \sin \phi=0, \quad T_{1}-F_{2} \sin \phi-T_{2} \cos \phi=0,
$$

which in turn leads to

$$
T_{1}=\left(F_{1} \cos \phi+F_{2}\right) / \sin \phi, \quad T_{2}=\left(F_{1}+F_{2} \cos \phi\right) / \sin \phi .
$$

Since $F_{1}$ and $F_{2}$ are shear forces at point $\mathbf{B}$, they may be written as

$$
F_{1}=-Q \kappa^{2} S \partial^{3} \xi /\left.\partial x^{3}\right|_{x=L}, \quad F_{2}=-Q \kappa^{2} S \partial^{3} \zeta /\left.\partial y^{3}\right|_{y=1},
$$

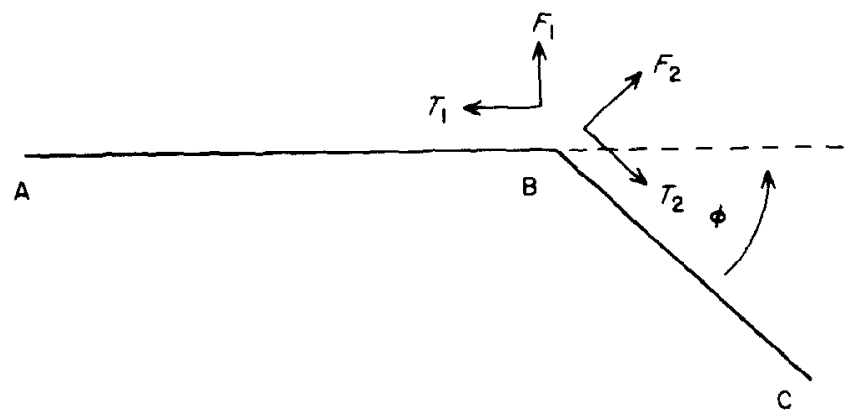

Figure 4. Force balance at the kink. 
where the approximation that $\xi$ and $\zeta$ are the linear modes previously derived has been used. One can see from equations (13)-(16) that $T_{1}$ and $T_{2}$ are of first order in $\xi$ and $\zeta$; however their inclusion in equations (1) and (2) is such that the forcing terms $T_{1} \partial^{2} \xi / \partial x^{2}$ and $T_{2} \partial^{2} \zeta / \partial y^{2}$ are second order. For a single mode $(\xi, \zeta)$ of frequency $\omega$ on the bar, it is clear from expressions (13) and (14), taken with expressions (15) and (16), that these forcing terms have frequency $2 \omega$.

The second non-linearity to be considered arises from unbalanced moments around point $\mathrm{B}$. This time the non-linear driving mechanism is derived independently of the linear modes and applied later to the linear solution. Equations (1), (2), (4) and (6) still hold but the boundary conditions at point $B$ are now written as the full equations (8) to include the second-order change in length of the bar that was neglected in the linear case. These length changes are given by

$$
\Delta=\int_{0}^{L}\left[1+(\partial \xi / \partial x)^{2}\right]^{1 / 2} \mathrm{~d} x-L
$$

for section $\mathrm{AB}$ and

$$
\delta=\int_{0}^{l}\left[1+(\partial \zeta / \partial y)^{2}\right]^{1 / 2} \mathrm{~d} y-l
$$

for BC. Thus for a bar vibrating with frequency $\omega$, point B undergoes both a static and an oscillatory $(2 \omega)$ displacement. Figure 2 illustrates the oscillatory displacements around the static displacement of $B$. The static displacement may be neglected as it is not significant in these investigations, so that the non-linear displacements $\xi_{2 \omega}^{\prime}, \zeta_{2 \omega}^{\prime}$ can be written as

$$
\xi_{2 \omega}^{\prime}=Z_{1}(x) \cos 2 \omega t, \quad \zeta_{2 \omega}^{\prime}=Z_{2}(y) \cos 2 \omega t
$$

It should be noted that $\xi_{2}^{\prime}$ and $\zeta_{2}^{\prime}$ are the solutions to equations (1) and (2) but with different boundary conditions at point $B$ from those of the linear solutions. Hence, neglecting damping, one may write

$$
\begin{gathered}
Z_{1}=e \cos \alpha_{2} x+f \cosh \alpha_{2} x, \\
Z_{2}=E\left(\sin \alpha_{2} y-\sinh \alpha_{2} y\right)+F\left(\cos \alpha_{2} y-\cosh \alpha_{2} y\right) .
\end{gathered}
$$

As has been said, point B may no longer be taken to be fixed as in the linear case. However one may approximate by assuming that the angle $\phi$ remains unchanged and that there are no linear modes of frequency $2 \omega$ initially present. For the linear modes one would have had

$$
\xi(L)=\zeta(l)=0, \quad \partial \xi /\left.\partial x\right|_{x=L}=-\partial \zeta /\left.\partial y\right|_{y=l}
$$

whereas for the non-linear mode, to a first approximation,

$$
\partial \xi^{\prime} /\left.\partial x\right|_{x=L}=0, \quad \partial \zeta^{\prime} /\left.\partial y\right|_{y=1}=0 .
$$

Equation (24) relates $e$ to $f$ and $E$ to $F$. Furthermore the geometry of Figure 2 gives, at point $\mathrm{B}$, since $Z_{1}$ and $Z_{2}$ as drawn are negative,

$$
Z_{1}=-\delta \sin \phi+Z_{2} \cos \phi, \quad Z_{2}=-\Delta \sin \phi+Z_{1} \cos \phi .
$$

Together with equations (19)-(22) these are now sufficient conditions to express the amplitude $e$ of the non-linear $2 \omega$ mode in terms of the amplitude $a$ of the linear $\omega$ mode initially present on the bar. 
The forcing mechanism at point $B$ that is being considered at present is a result of unbalanced bending moments $M_{1}$ and $M_{2}$ on the two sections of the bar, where

$$
M_{1}=-Q S \kappa^{2} \partial^{2} \xi_{2 \omega}^{\prime} / \partial x^{2}, \quad M_{2}=-Q S \kappa^{2} \partial^{2} \zeta_{2 \omega}^{\prime} / \partial y^{2}
$$

The total unbalanced moment $M=M_{1}-M_{2}$ then appears as a driving term in equations (1) and (2) of the form

$$
M(\mathrm{~d} / \mathrm{d} x) \delta(x-L), \quad M(\mathrm{~d} / \mathrm{d} y) \delta(y-l),
$$

where $\delta(x)$ is the Dirac delta function. The frequency of this driving term is, once again, $2 \omega$.

The length changes $\Delta$ and $\delta$ of equations (18) and (19) lead to a further non-linearity which requires identification, though it will not be investigated in detail. As well as a concentrated moment of frequency $2 \omega$ at the kink, these contractions give rise to tension forces $T_{1}^{\prime}$ and $T_{2}^{\prime}$ in the two parts of the bar, also at a frequency $2 \omega$. Indeed the tension $T_{1}^{\prime}$ would be present for the same reason in a straight bar with rigidly clamped ends. The driving terms arising from this tension have the forms

$$
T_{1}^{\prime} \partial^{2} \xi / \partial x^{2}, \quad T_{2}^{\prime} \partial^{2} \zeta / \partial y^{2}
$$

and are of third order and frequency $3 \omega$. Their importance can be expected to be less than that of second order terms at frequency $2 \omega$ already discussed.

\section{SOLUTION}

The equations of motion (1) and (2) must now be modified to include the non-linear forcing terms specifically. To do this one can suppose that $u_{n}(t) \psi_{n}(x)$ represents the normal modes over the whole range of $x$ from 0 to $L+l$, with $\psi_{n}(x)=\xi_{n}(x)$ for $0 \leqslant x \leqslant L$ and $\psi_{n}(x)=\zeta_{n}(L+l-x)$ for $L \leqslant x \leqslant L+l$. One can further suppose that the $\psi_{n}$ are normalized over this whole range-they are, of course, orthogonal. The problem in hand is to examine the behaviour of a mode $p$ of frequency $\omega_{p}$ and small amplitude in the presence of a large-amplitude mode $m$, where $\omega_{p} \approx 2 \omega_{m}$. Equations (1) and (2) for mode $p$ can then be written in combination as

$$
\rho \psi_{p} \frac{\partial^{2} u_{p}}{\partial t^{2}}=-Q \kappa^{2} u_{p} \frac{\partial^{4} \psi_{p}}{\partial x^{4}}-D \psi_{p} \frac{\partial u_{p}}{\partial t}+\frac{T(m)}{S} u_{m} \frac{\partial^{2} \psi_{m}}{\partial x^{2}}+M(2 m) \frac{\mathrm{d}}{\mathrm{d} x} \delta(x-L),
$$

where $T(m)=T_{1}$ for $0 \leqslant x \leqslant L$ and $T_{2}$ for $L \leqslant x \leqslant L+l . T(m)$ and $u_{m}$ vary with frequency $\omega_{m}$ and $M(2 m)$ with frequency $2 \omega_{m}$.

Now multiplying both sides of (31) by $\psi_{p}(x)$ and integrating over $0 \leqslant x \leqslant L+l$, in view of the orthonormality, gives

$$
\frac{\mathrm{d}^{2} u_{p}}{\mathrm{~d} t^{2}}+\omega_{p}^{2} u_{p}=-\frac{D}{\rho} \frac{\mathrm{d} u_{p}}{\mathrm{~d} t}+\frac{u_{m}}{\rho S}\left[T_{1}(m) I_{1}+T_{2}(m) I_{2}\right]+\left.\frac{M(2 m)}{\rho} \frac{\mathrm{d} \psi_{p}}{\mathrm{~d} x}\right|_{x=L},
$$

where

$$
I_{1}=\int_{0}^{L} \Psi_{p}(x) \frac{\mathrm{d}^{2} \psi_{m}(x)}{\mathrm{d} x^{2}} \mathrm{~d} x, \quad I_{2}=\int_{L}^{L+1} \psi_{p}(x) \frac{\mathrm{d}^{2} \psi_{m}(x)}{\mathrm{d} x^{2}} \mathrm{~d} x .
$$

The second and third terms on the right side of equation (32) are forcing terms, both with frequency $2 \omega_{m}$. They vanish in the linear approximation.

The relative importance of the contribution made by the tension terms $T$ and moment term $M$ in equation (32) is not immediately clear and may vary from case to case. For the relatively thin bar examined experimentally, theory suggests that the tension terms are clearly dominant and indeed the moment term is so small it can be neglected. 
If one had been interested in the third order $3 \omega$ terms set out in expression (30) then these would enter equation (32) similarly as $\left[T_{1}^{\prime} I_{1}+T_{2}^{\prime} I_{2}\right]$. Since the integral $I_{1}$ does not vanish even for a rigidly clamped bar, this mode coupling mechanism is always present. This is in counter-distinction from the case of a rigidly supported string, for which the equivalent integral vanishes identically.

The differential equation (32) for the $p$ th mode has the general form

$$
\mathrm{d}^{2} u_{p} / \mathrm{d} t^{2}+\omega_{p}^{2} u_{p}=g(t)
$$

where $g$ contains both damping and forcing terms. If one writes

$$
u_{p} \equiv a_{p} \sin \left(\omega_{p} t+\theta_{p}\right),
$$

where both $a_{p}$ and $\theta_{p}$ are slowly varying functions of time, then equation (34) is in the standard form for treatment by the method of slowly varying parameters $[6,7]$. The procedure is now analogous to that used in the previous analysis of the string [5] and the resulting equations are

$$
\begin{gathered}
\left\langle\dot{a}_{p}\right\rangle=\beta_{m} a_{m}^{2} \cos \left[\left(2 \omega_{m}-\omega_{p}\right) t+2 \theta_{m}-\theta_{p}\right]-a_{p} / \tau_{p}, \\
\left\langle\dot{\theta}_{p}\right\rangle=\beta_{m}\left(a_{m}^{2} / a_{p}\right) \sin \left[\left(2 \omega_{m}-\omega_{p}\right) t+2 \theta_{m}-\theta_{p}\right],
\end{gathered}
$$

where the mode coupling coefficient $\beta_{m}$ follows from equation (32) and it has been assumed that $\omega_{p} \approx 2 \omega_{m}$.

Since all that is driving mode $p$, when it is assumed to start from zero amplitude, is the second harmonic of mode $m$, and since $a_{m} \gg a_{p}$ has been assumed, one can write

$$
\theta_{m}=\text { const, } \quad \dot{\theta}_{p}=2 \omega_{m}-\omega_{p},
$$

and then equation (37) gives

$$
2 \omega_{m}-\omega_{p}=\beta_{m}\left(a_{m}^{2} / a_{p}\right) \sin \left(2 \theta_{m}-\theta_{p}^{0}\right),
$$

where $\theta_{p}^{0}$ is the value of $\theta_{p}$ at $t=0$. Inserting expressions (38) and (39) in equation (36) then gives

$$
\left\langle\dot{a}_{p}\right\rangle=\beta_{m} a_{m}^{2}\left\{1-\left(2 \omega_{m}-\omega_{p}\right)^{2} a_{p}^{2} / \beta_{m}^{2} a_{m}^{4}\right\}^{1 / 2}-a_{p} / \tau_{p} .
$$

Clearly the rate of increase of $a_{p}$ is greatest if the resonance condition $\omega_{p}=2 \omega_{m}$ is closely satisfied, as indeed one should expect.

In the experiment to be described, the amplitude of mode $m$ is initially large and then decays exponentially, while the amplitude of mode $p$ is initially nearly zero. Equation (40) then predicts that $a_{p}$ will rise to a maximum, which depends on the behaviour of $a_{m}$, and then decay towards zero.

This behaviour can be made explicit for the simple case in which the decay times $\tau_{m}$ and $\tau_{p}$ are constants and $\omega_{p}$ is exactly equal to $2 \omega_{m}$. Integration of equation $(40)$ for the case of no initial excitation of mode $p$ then gives

$$
a_{p}(t)=\beta_{m}\left(a_{m}^{0}\right)^{2}\left(\frac{\tau_{m} \tau_{p}}{\tau_{m}-2 \tau_{p}}\right)\left(\mathrm{e}^{-2 t / \tau_{m}}-\mathrm{e}^{-t / \tau_{p}}\right),
$$

where $a_{m}^{0}$ is the initial amplitude of mode $m$. In the more general case, integration of (37) and (40) can give beat-like phenomena superposed on a curve of the same general shape as given by (41).

Another simple case that can be solved explicitly is that of continuous excitation of mode $m$ to amplitude $a_{m}^{0}$. This is equivalent to letting $\tau_{m} \rightarrow \infty$, so that

$$
a_{p}(t)=\beta_{m}\left(a_{m}^{0}\right)^{2} \tau_{p}\left(1-\mathrm{e}^{-t / t_{p}}\right) .
$$




\section{EXPERIMENT}

Since mode conversion is significant only when one normal mode has nearly twice the frequency of another, the dimensions of the bar are important. For a galvanized steel bar of rectangular cross-section and thickness $0.6 \mathrm{~mm}$, and length $30 \mathrm{~cm}$ between $B$ and $B^{\prime}$ (refer to Figure 1), the lengths of ends $B C$ and $B^{\prime} C^{\prime}$ necessary to achieve an appropriate resonance can be calculated. Figure 3 shows how the resonant frequencies of the symmetric modes vary with change in length of the bent ends. The figure shows that, for such a bar, if the bent ends are about $9 \mathrm{~cm}$ in length, then the third symmetric mode $\left(\omega_{3}\right)$ will have twice the frequency of the second symmetric mode $\left(\omega_{2}\right)$.

A bar of these dimensions was clamped between two tilting vices and the admittance characteristic at its centre point A determined with a Brüel \& Kjaer impedance head type 8001 in conjunction with an integrator and a dual channel FFT spectrum analyzer. The length of the bent ends was varied until $\omega_{3}$ was closely equal to $2 \omega_{2}$, the final length being approximately $8.8 \mathrm{~cm}$. The admittance curve measured is shown in Figure 5 together with the admittance calculated for such a bar by using the linear theory and neglecting damping.

The first check for the behaviour described in equation (40) was to shake the bar, at a frequency equal to $\omega_{2}$, on a node of the third node, and to use a subminiature accelerometer positioned at the centre point $A$ of the bar to measure the amount of third

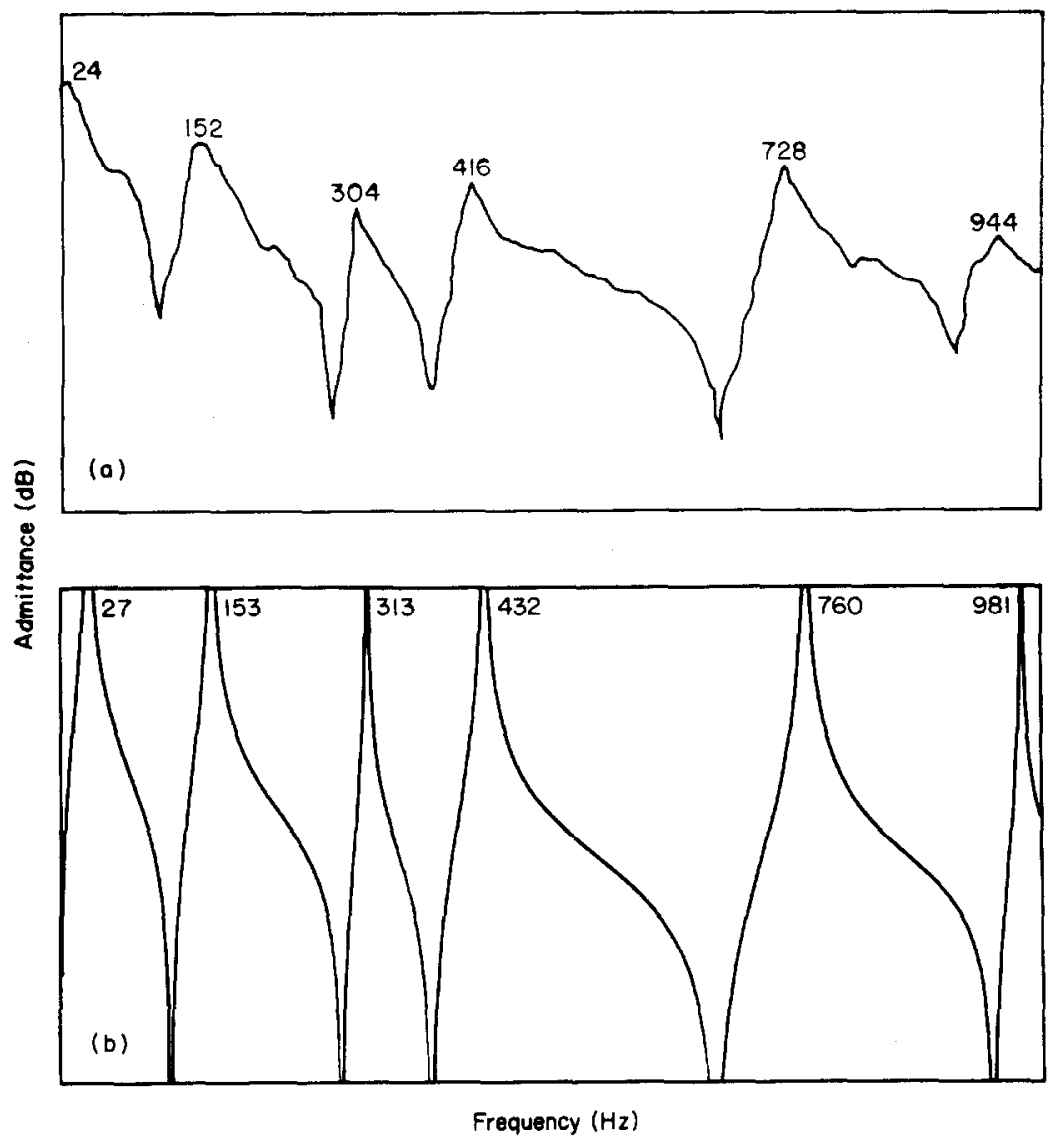

Figure 5. Mechanical admittance at the centre point $A$ of a symmetrically kinked bar (a) as measured, and (b) as calculated. 
mode generated. Positioning of the shaker on this node was to ensure that any $\omega_{3}$ component present as distortion in the shaker output was of negligible consequence to the result. It also served the purpose of ensuring that the only point "clamped" into vibrating at $\omega_{2}$ only by the presence of the shaker was a node of $\omega_{3}$.

The result of this measurement is shown in Figure 6 and has the form

$$
a_{3} \approx 250 a_{2}^{2}
$$

where $a_{3}$ and $a_{2}$ are both measured in metres. This agrees with the limiting form of equation (42) as $t \rightarrow \infty$. As will be presently discussed, the bar has a value of $\tau_{3}$ equal to about $0.25 \mathrm{~s}$ so that the coupling coefficient $\beta_{2}$ has a value of about $1000 \mathrm{~m}^{-1} \mathrm{~s}^{-1}$ for this particular bar. The actual value calculated from the rather involved expression in equations (32) and (33) is about $2600 \mathrm{~m}^{-1} \mathrm{~s}^{-1}$. In view of the approximations in the theory, this agreement should be regarded as moderately satisfactory.

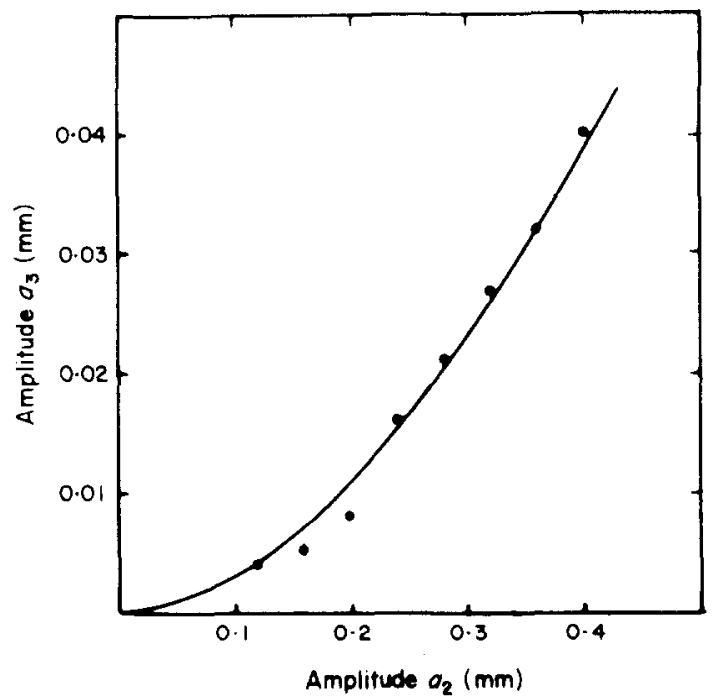

Figure 6. Measured amplitude $a_{3}$ of the third mode as a function of the amplitude $a_{2}$ of the second mode for a kinked bar shaken at a node of the third mode. The curve is $a_{3}=$ const $\times a_{2}^{2}$.

The theory was further examined in a second experiment, in which the bar was struck with a weighted piano hammer on a node of the third mode, the objective being to excite the bar into a vibration that initially excluded this mode and to observe the mode's subsequent amplitude. The vibration signal from a subminiature accelerometer, positioned as before at the centre of the bar, was recorded on a Nagra IV tape recorder and later analyzed with an appropriate filter to determine the time behaviour of both the second and third modes. The analysis showed that the second mode decayed more or less exponentially in time with $\tau_{2}=0.26 \mathrm{~s}$ while the third mode grew from near zero to a maximum in a time of order $0.1 \mathrm{~s}$ before decaying slowly to zero. A typical trace is shown in Figure 7. The peak amplitude reached by the missing third mode, and the time between the hammer blow and this peak, were both plotted as functions of $a_{2}^{0}$, the initial amplitude of the second mode. These measurements are shown in Figure 8 along with the behaviour predicted by theory.

Figure 9 shows the calculated behaviour of the third mode amplitude when the bar is hit so as to make its initial value zero. Comparison with Figure 7 shows that the general predictions of the theory are qualitatively similar to the experimental behaviour. 


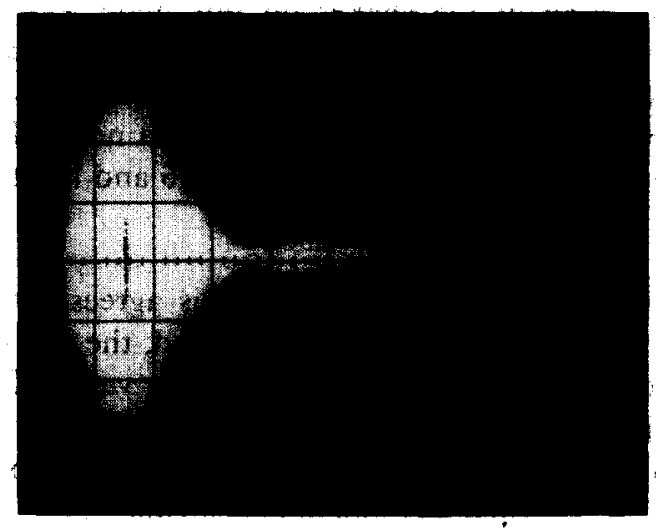

Figure 7. Oscilloscope trace of the behaviour of the third mode amplitude with time when a kinked bar is excited by a hammer blow close to a node for this mode. Total tracelength is $1 \mathrm{~s}$.

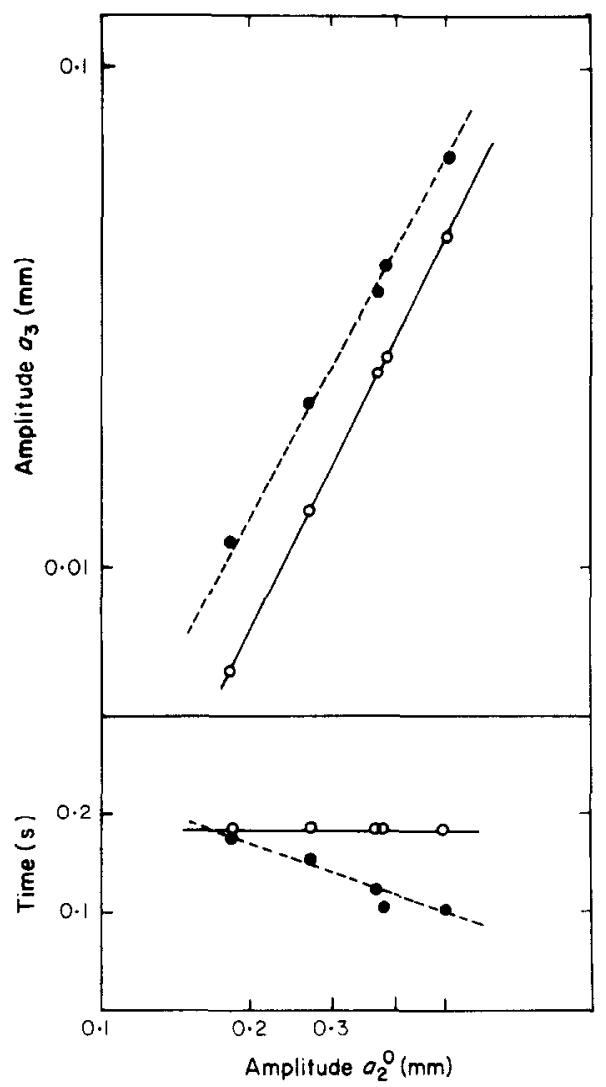

Figure 8. Calculated (open circles) and measured (filled circles) dependence of third mode peak amplitude $a_{3}$ and time delay on the initial amplitude $a_{2}^{0}$ of the second mode for a kinked bar struck at a node of the third mode. 


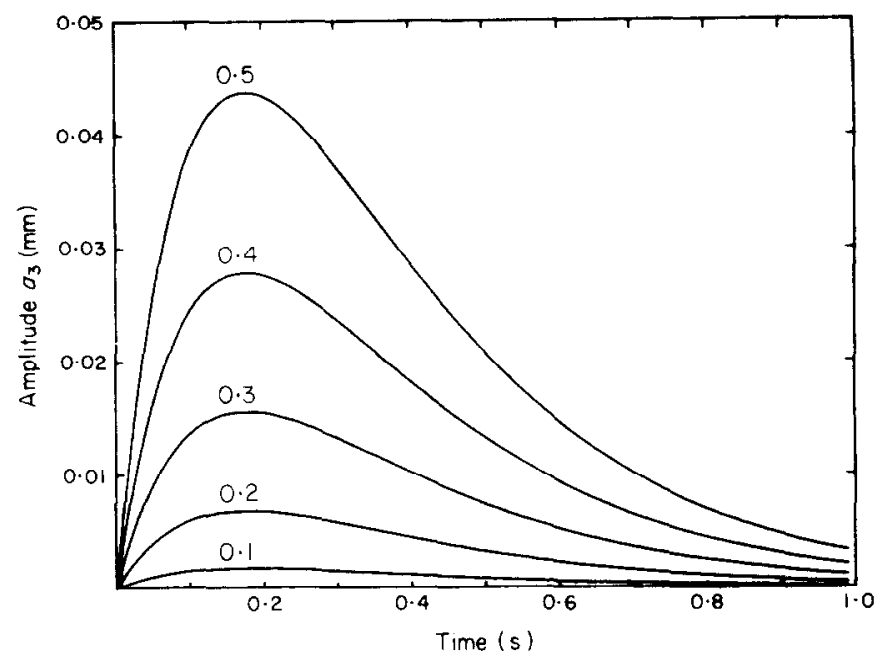

Figure 9. Calculated behaviour of the third mode amplitude $a_{3}$ as a function of time for different initial amplitudes of the second initial mode excitation (shown as a parameter), with simple linear damping assumed.

For a more quantitative assessment of the theory one must turn to Figure 8 which shows the predicted maximum amplitude and time to reach that amplitude plotted alongside the experimental data. It must be noted however that the theory relies on the measurement of the decay constants $\tau_{2}$ and $\tau_{3}$, which were determined in a separate free-decay experiment. These in turn rely on there being no interaction between modes while these parameters are being measured. While the second mode decayed exponentially with time with $\tau_{2}=0.26 \mathrm{~s}$, the decay time of the third mode appeared to change with amplitude, becoming longer for smaller amplitude. Clearly with several modes present on the bar one would expect interaction terms which would affect the apparent damping. For the purpose of the above experiment it was considered adequate to take an average decay rate of $\tau_{3}=0.25 \mathrm{~s}$. This approximation may however explain in part the small discrepancies between theory and experiment shown in Figures 7-9.

The approximate agreement in slope and magnitude between the experimental and theoretical curves in Figure 8 supports the general correctness of the behaviour described by equation (41). The value of $\beta_{2}$ implied by the experimental results is about $4000 \mathrm{~m}^{-1} \mathrm{~s}^{-1}$ which is larger than the calculated value of $2600 \mathrm{~m}^{-1} \mathrm{~s}^{-1}$. In view of the behaviour of $\tau_{3}$ this agreement is reasonably good, but the origin of the deviation from the value given by the steady state experiment is not clear.

\section{CONCLUSIONS}

The semi-quantitative agreement between theory and experiment gives confidence that the major physical mechanisms have been identified which are responsible for mode coupling and transfer of energy between harmonically related modes on a symmetrically bent bar. While other effects could enter for bars with much more complicated geometry, the mechanism examined here can be expected to be present in all cases. Indeed, it is tempting to speculate that such a mechanism may be largely responsible for the transfer cascades observed in the large-amplitude vibration of symmetrically flanged gongs.

More generally, mode coupling and energy transfer of this type would be expected to occur in all plate-like systems having a sharp kink or crease. Whether or not a significant amount of energy is transferred to the higher mode will depend upon the extent to which 
there is agreement between the frequency of a higher mode and that of the second harmonic of some mode that is being driven to large amplitude.

\section{REFERENCES}

1. A. V. SRINIVASAN 1966 International Journal of Nonlinear Mechanics 1, 179-199. Nonlinear vibrations of beams and plates.

2. J. G. EISLEY 1964 Zeitschrift für angewandte Mathematik und Physik 15, 167-175. Nonlinear vibrations of beams and rectangular plates.

3. N. H. FletChER 1985 Journal of the Acoustical Society of America 78, 2069-2093. Nonlinear frequency shifts in quasispherical-cap shells: Pitch glide in Chinese gongs.

4. T. D. Rossing and N. H. Fletcher 1982 Bulletin of the Australian Acoustical Society 10, 21-26. Acoustics of a tamtam.

5. K. A. LegGe and N. H. Fletcher 1984 Journal of the Acoustical Society of America 76, 5-12. Nonlinear generation of missing modes on a vibrating string.

6. N. H. Bogoliubov and Y. A. Mitropolsky 1961 Asymptotic Methods in the Theory of Non-linear Oscillations. Delhi: Hindustan Publishing Company and New York: Gordon and Breach. See pp. 39-55.

7. P. M. MORSE and K. U. INGARD 1968 Theoretical Acoustics. New York: McGraw-Hill. See pp. 838-839. 